\title{
本科实验课程中的主观能动性激励方法
}

\author{
王奇峰 $1,{ }^{*}$ ，项金宝 ${ }^{2}$ \\ 1吉林大学化学学院, 长春 130012 \\ 2 吉林大学药学院, 长春 130021
}

摘要: 本科生实验课程是对理论知识进行实践的重要环节。为提高本科生在实验过程中对实验理论和技能的理性认 识, 增强自主学习的意识和兴趣, 提出了 “理论质疑” 和 “步骤调整” 的方法, 以激励学生在实验过程中能更好地 发挥创造性思维, 提升实验操作能力。该方法经过作者在指导本科生基础有机化学实验的过程中证明, 学生在实验 过程中的积极性得到明显提升, 增强了对所学理论知识的认识和理解, 更善于提出新的科学问题, 能对实验方法的 合理性和继续改进提出新的见解, 理解了 “尽信书不如无书” 的道理。

关键词: 有机化学; 实验; 创造性; 本科教学

中图分类号: G64; O6

\section{Stimulation of Subjective Initiative in Undergraduate Laboratory Course}

\author{
Qifeng Wang ${ }^{1, *}$, Jinbao Xiang ${ }^{2}$ \\ ${ }^{1}$ College of Chemistry, Jilin University, Changchun 130012, China. \\ ${ }^{2}$ School of Pharmaceutical Sciences, Jilin University, Changchun 130021, China.
}

\begin{abstract}
Undergraduate laboratory course is important for understanding the theoretical knowledge. In order to improve undergraduates' rational understanding of experimental theory and skills, and enhance their awareness and interests in autonomous learning, this paper puts forward the methods of "theory query" and "step adjustment" to encourage students to give a full play to their creative thinking and improve their lab skill. This method has been practiced in the organic chemistry laboratory under the guidance of the authors. As a result, the enthusiasm of students in the experimental process has been significantly improved; more scientific problems and new ideas have been put forward for the rationality and continuous improvement of the experimental method, and the truth that "it is better to believe than not to have books" has been understood.
\end{abstract}

Key Words: Organic chemistry; Experiment; Creativity; Undergraduate teaching

本科生的学术能力和素养的提升包括理论知识的教授和实验操作能力的培养 ${ }^{[1]}$ 。教育部长期以 来非常关注本科生的实验能力培养, 并提出了 “科教结合协同育人行动计划” “基础学科拔尖学生培 养试验计划” 等, 为本科生的实验创新能力提升提供支持。同时, 大学教师也长期探索本科生的实 验教学方法, 以让本科生获得更高的实验能力 ${ }^{[2,3]}$ 。化学领域作为一门重要的实验学科, 其对本科生 的实践操作能力要求更为严格, 这一方面关乎学生对理论知识的掌握和运用, 旨在能够使学生在实

收稿: 2021-08-04; 录用: 2021-08-30; 网络发表: 2021-09-17

“通讯作者, Email: wangqifeng@jlu.edu.cn

基金资助：吉林大学本科教学改革研究项目(院培育项目) (2021JG02); 吉林大学化学自主双一流建设基金 
际的化学工作中合理地设计实验条件和路线, 以更为简洁和高效的方式完成工作任务; 另一方面也 是对实验安全以及操作人员安全的重要保障, 为将来的化学相关事业的发展提供最初步的安全保障。

在目前国内的高校中, 基础有机化学实验是一门非常普遍和重要的教学科目, 其教学方式通常 以教学参考书为指南, 每学期从中选取 10-12次的基础实验项目, 按照课本中的实验步骤进行教学活 动。在这种教育模式下, 作者在长期的实践教学过程中发现, 学生通常不能理解每一步实验中所采 用的数据和信息的来源、注意事项的理由, 以及理论上合理的实验结果, 更不明白实验按照该步骤 进行的科学意义。因此, 学生在一学期甚至一学年实验课程学习之后并不能合理地设计和操作实验, 包括实验步骤安排、试剂用量、检测方法等, 甚至出现潜在危险都浑然不知。针对这种现象, 不同 高校的教师曾提出多种解决方案, 包括 “做好实验预习” [4]、“培养实验习惯” [5]、或者 “对本科生 开放高级实验室” [6,7]等方法, 但情况并没有因此获得有效的改善, 抄写实验报告反而成为学生的负 累, 进入高级实验室成为本科生获取学分的捷径。为改善和解决此类相关问题, 作者在实验指导过 程中提出了 “理论质疑” 和 “步骤调整” 的方法, 以提高本科生在学习过程中的主观能动性, 激发 思考问题的兴趣, 并锻炼应付突发情况的能力。通过实践发现, 这两种方法对于改善学生主观能动 性具有非常明显的效果, 学生会由此对参考书中的步骤和数据进行更为深刻的理解, 进而以更为简 洁高效的方式完成实验工作，体现出了“尽信书不如无书”的价值。

\section{1 “理论质疑” 方法的提出与实施}

“理论质疑” 法是作者依据自然辩证法中 “否定之否定” 规律针对学生填鸭式教学提出的一种 方法, 旨在让学生对参考书中的实验步骤要求提出合理的质疑, 并在实验可控的条件下对质疑的内 容进行探索性尝试, 根据观察到的实验现象阐述参考书中实验步骤的合理性, 即对实验步骤要求首 先进行 “否定” , 根据实验现象决定对 “否定” 的结果进行 “肯定” 或 “否定” , 最终达到对实验 步骤更为准确和合理的认识。

以《基础化学实验》 ${ }^{[8]}$ 中 “乙醇的蒸馏” 实验为例。该实验处于本科生进行基础有机实验的最初 阶段, 学生对基础理论与实验实际情况的结合充满了好奇。在实验步骤中关于现象的描述如下: “当 瓶内只剩下少量液体时, 若维持原来的加热速度, 温度计的读数会突然下降, 即可停止蒸馏。” 这 段文字的表达, 是指当主馏分蒸馏完毕之后, 剩下的高沸点后馏分由于温度不够而不能挥发, 所以 温度计的液泡检测不到任何蒸汽的温度, “温度计的读数会突然下降”, 也意味着主馏分蒸馏完成, 实验结束。

尽管这是一个正确的现象描述, 但在实际实验过程中, 作者通过多年的观察, 发现只有较低比 例的学生会观察到该现象, 而大多数学生会观察到温度出现急剧上升的 “反常” 现象, 这些学生对 于自己遇到的与课本描述不同的实验现象产生迷茫或者产生实验失败的恐惧心理。为此, 作者尝试 鼓励学生对书本文字进行 “理论质疑” 。作者对学生提出以下问题: “已知乙醇的沸点是 $78^{\circ} \mathrm{C}$, 后 馏分的沸程在 $100-110^{\circ} \mathrm{C}$, 如果反应瓶的实际加热温度达到 $120^{\circ} \mathrm{C}$, 请问实验过程中是否会观察到 书本所说的温度突然下降的现象? ” 针对这个问题, 学生经过激烈的讨论, 统一认为不能下降, 而 是应该上升, 进而学生开始重新审视课本的描述为什么和他们所观察到的实验现象不一致, 经过 “理 论质疑” 他们进一步明白, 对加热温度非常精确的控制, 才有机会观察到 “读数会突然下降” 的情 况, 而当温度设置过高之后, 高沸点后馏分的挥发, 引起了温度的上升, 而这同样是主馏分蒸馏完 毕的现象。事实上, 在环境温度、保温条件、加热器功率、加热模块与烧瓶的距离和接触面积等诸 多复杂因素的综合影响之下, 学生非常难以控制实际的加热温度, 通过温度控制蒸馏速度也很困难, 温度设置偏高是非常常见的现象, 通过对课本所描述的现象的质疑, 进一步增强了学生的实验兴趣, 也使他们能更深刻地理解实验原理, 达到实验的目的。

再以《基础化学实验》 ${ }^{[8]}$ 中实验 “2-甲基-2-己醇” 为例, 该实验中有如下步骤描述：“ $\mathrm{A}$. 与丙 酮反应: 将制好的格氏试剂在冰水浴冷却和摚拌下, 自滴液漏斗中滴入 $5 \mathrm{~mL}$ 丙酮和 $8 \mathrm{~mL}$ 无水乙醚的 
混合液, 控制滴加速度, 勿使反应过于猛烈。加完后, 在室温继续搅拌 $15 \mathrm{~min}$ 。溶液中可能有白色粘 稠状固体析出。” 以及 “B. 分解格氏试剂: 将反应瓶在冰水浴冷却和摚拌下, 自滴液漏斗分批加入 $50 \mathrm{~mL} \mathrm{10 \%}$ 硫酸溶液, 分解产物(开始滴入宜慢, 以后可逐渐加快)。”

在步骤A中提到 “滴入 $5 \mathrm{~mL}$ 丙酮”, 学生按照 “控制滴加速度, 勿使反应过于猛烈” 的实验要 求, 以将近 30 秒/商的滴加速度滴入丙酮, 做到了 “勿使反应过于猛烈”。然而, 学生并不理解怎么 达到合理的滴加速度, 也不理解 “反应过于猛烈” 所描述的实际意义是什么, 只能在实验台前消磨 时间。按照每一滴 $0.02 \mathrm{~mL}$ 计算, 滴加 $5 \mathrm{~mL}$ 需要 $2 \mathrm{~h}$ 。基于这种情况, 作者对学生提出如下问题: 1) 该 反应发生的机理是什么, 应该出现什么实验现象? 2) 反应为什么不能过于猛烈, 猛烈会导致什么后 果? 其中第二个问题即是对理论的质疑。学生在经过认真思考和讨论之后, 对第一组问题都能够正 确回答: “反应遵循亲核加成的机理, 有放热的现象”, 但对于第二组问题, 学生基本无法正确回 答。这种现象一方面说明学生不明白危险产生的原因和危险的表现形式, 而只能按照因噎废食的状 态循规蹈矩; 另一方面, 一旦出现相关危险, 学生由于不知道危险产生的原因, 也不知道该怎么应 对, 可能会产生更严重的后果。为此, 安排学生首先保证有充足的冰水浴冷却以及完善的回流装置, 在能够保证实验安全的情况下, 要求学生将滴加速度加快, 在此基础上观察反应猛烈的状态。

对于这种实验方式的改变, 学生产生了极大的探索性兴趣。通过滴加速度的提升, 学生观察到 反应由于放热而产生了明显的沸腾现象, 冷凝装置出现了回流, 由于冰水浴的存在, 反应并没有出 现危险和无法控制的猛烈状态。通过这种操作, 反应时间至少缩短至预计时间的 $1 / 2$, 且实验能够顺 利完成。基于这个操作, 与学生经讨论之后得出结论: 参考书中要求的 “勿使反应过于猛烈” 旨在 防止反应放热造成温度过高, 溶剂回流无法控制, 继而出现危险。在本实验中, 由于反应试剂用量 比较小, 且有冰水浴控制温度, 以及回流装置控制回流, 反应不会出现无法控制的局面; 如果在反 应试剂用量过大、甚至工业级小试以上规模的情况下, 或冷却以及回流装置缺失的情况下, 反应一 定需要控制滴加速度, “勿使反应过于猛烈”。

在步骤B的操作中, 课本同样要求了 “开始滴入宜慢, 以后可逐渐加快” 的操作规程, 由于对 “理 论质疑” 法的准确理解, 学生在滴加硫酸溶液的过程中充分考虑了加入硫酸由慢到快的缘由, 因此 能根据反应放热所表现的状态, 灵活地控制滴加速度, 游刃有余地完成了实验内容, 且极大提高了 实验效率。

\section{2 “步骤调整” 方法的提出与实施}

“步骤调整” 是作者根据现有反应路线设计的合理性提出的一种尝试性改变方法的策略, 一方 面能增加学生的创新性体验, 另一方面能让学生更加理解原有实验步骤的设计合理性。

以《有机化学实验》 ${ }^{[9]}$ 中 “正溴丁烷” 为例, 其中部分反应步骤描述为: “由于无机盐水溶液有 较大的相对密度, 不久会分出上层液体即是正溴丁烷。回流 $40 \mathrm{~min}$ 。待反应液冷却后, 移去冷凝管, 加上蒸馏头, 改为蒸馏装置, 蒸出粗产物正溴丁烷。”

该步骤要求学生在反应生成的正溴丁烷和硫酸氢钠水溶液分层之后, 将混合物通过蒸馏操作将 产物正溴丁烷和水蒸馏出来, 之后将混合液进行分液后处理。针对这个步骤, 作者通过对本科生多 年的观察发现, 从未有本科生对该步骤有过任何异议, 即为什么在反应瓶中产物已经和水溶液分层 (如图1(a)所示), 却不能直接分液, 而是采用繁琐的蒸馏操作? 对于此操作, 作者要求学生对上述问 题进行 “理论质疑”。非常有趣的是, 对于这个提问, 学生会突然意识到这个问题非常有价值, 但经 过长时间的激烈探讨和思索, 却无法给出合理答复, 并一致认为直接分液是非常值得尝试的一种方 案。由于实验没有危险性, 作者果断让部分实验操作能力较好的学生进行了 “步骤调整”, 进行分液 操作, 学生因此而产生了极大的兴趣。

实验过程中, 学生发现, 在温热的条件下, 将反应后的混合液转移到分液漏斗之后, 需要快速 的分液操作。一旦冷却, 会有 $\mathrm{NaHSO}_{4}$ 固体析出, 造成分液困难。在此情况下, 需要在混合液中补加 


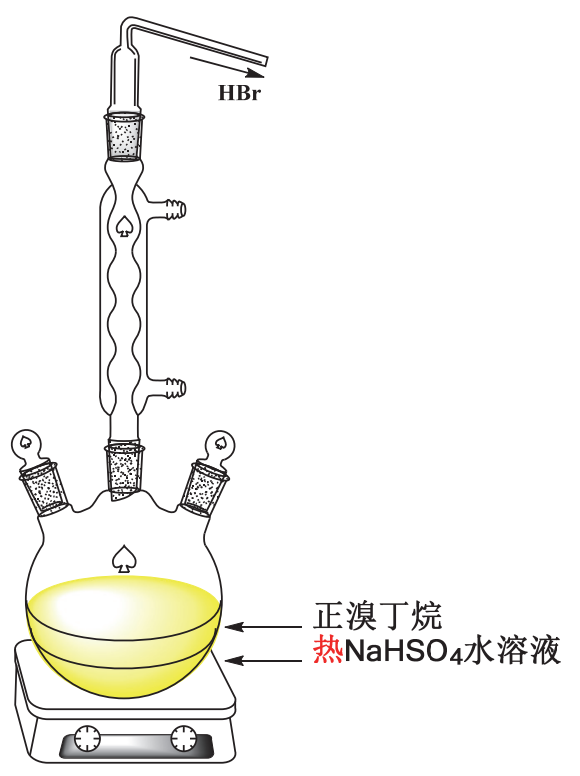

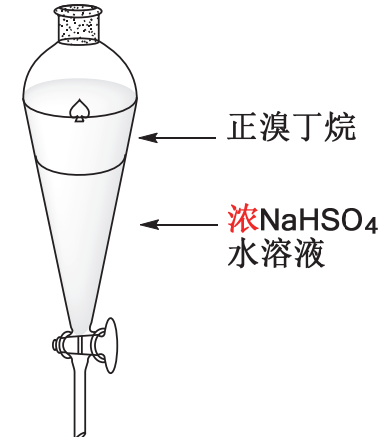

( b )

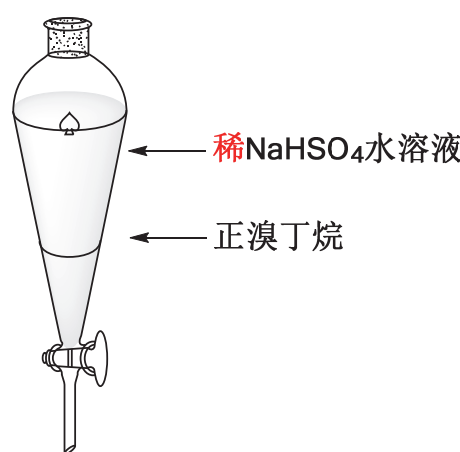

(c)

图1 正溴丁烷合成反应后静置状态以及转移至分液漏斗的分层状态

水, 以溶解析出的盐。当补加等体积的水时, $\mathrm{NaHSO}_{4}$ 能够溶解且溶液密度仍大于正溴丁烷, 此时正 澳丁烷在上层; 当补加二倍体积的水时, $\mathrm{NaHSO}_{4}$ 能够溶解且溶液密度小于水, 正澳丁烷转移至下层 (如图1(b)和(c)所示)。正是由于溶液密度变化的复杂性, 产物正溴丁烷在水相的上层或下层会不一 致, 所以为了简化这种操作, 课本中直接采用了蒸馏的方法, 以便于实验步骤的统一管理。参与 “步 骤调整” 实验的同学, 分别通过三个方案的实施, 均以非常高的产率获得了相应的产品, 且由于去 掉了蒸馏操作的搭建、蒸馏等步骤, 效率显著提高, 满意度和体验度极高。

再以《有机化学实验》[9]中 “甲基橙” 为例, 该实验在 “偶合” 步骤中要求 “在小雉形瓶内混合 $1.2 \mathrm{~g} \mathrm{~N}, \mathrm{~N}$-二甲基苯胺和 $1.0 \mathrm{~mL}$ 冰醋酸, 在不断搅拌下将此溶液慢慢加到上述冷却的重氮盐溶液中。” 该实验中由于重氮盐溶液不稳定, 始终要求保持低于 $5^{\circ} \mathrm{C}$, 因此操作难度较大。实验设计的原理, 是为了将这种不稳定的化合物尽快反应, 所以将 $N, N$-二甲基苯胺的醋酸盐滴加到大量的重氮盐中, 使其在反应初期能够尽可能地反应, 防止大量分解。但在实际操作过程中, 当苯胺醋酸盐加入到重 氮盐中之后, 随着反应的进行, 混合物黏度越来越大, 搅拌很难均匀, 放热过程复杂, 经常出现温 度急剧上升难以控制的现象, 其原因一方面是黏度过大导热不均匀, 另一方面, 是滴加的苯胺醋酸 盐未能均匀反应, 而累积到一定量之后与大量重氮盐突然反应, 最终导致大量放热。这种放热会加 速分解尚未反应的重氮盐, 造成产率下降的后果。

根据多年的教学经验, 作者在实验的开始阶段即与学生探讨本实验滴加的注意事项, 并向学生 提出这样一个问题: “如果我们将实验重新设计为将重氮盐滴加到 $N, N-$ 二甲基苯胺的醋酸盐中, 尽 管也会出现粘稠的现象, 但突然的放热只是已经滴加的重氮盐参与了反应, 完全不会分解尚未滴加 进去的重氮盐, 这样进行实验是否能获得更高的产率? ” 学生显然对这个问题没有心理准备, 但经 过激烈的讨论, 认为非常值得尝试。因此, 作者从踊跃报名的同学中选了部分同学进行 “步骤调整” 方案的实施。

实验过程中, 参与试验的同学发现, 由于是将重氮盐滴加到大量的 $N, N-$ 二甲基苯胺的醋酸盐溶 液中, 所以反应自开始阶段, 滴加进去的重氮盐就反应得非常彻底, 而在反应的中后期, 尽管粘稠 的混合物反应放热导致温度时而高于 $5^{\circ} \mathrm{C}$, 但在冷却后继续滴加重氮盐, 完全不影响实验结果, 而 最终参与 “步骤调整” 的同学都以更高的产率完成了实验, 极大提升了实验的兴趣和成就感。 


\section{3 结语}

作者在多年的教学过程中发现, 很多本科生有很强的创造性欲望, 但往往会受到一些传统教学 方法的限制, 没有机会发挥其相应的才能; 与此同时, 更多的本科生习惯于按照现有课本知识非常 顺从地学习和记忆, 很少质疑其可靠性。作者在科学研究探索过程中, 深知很多有机反应的理论知 识都是建立在假设的机理和探索性的方法上提出的, 需要根据 “否定之否定规律” 不断修正和改进, 这就需要有创造性欲望的青年一代勇于尝试, 勇于突破, 而不是单纯地接受填鸭式教育。作者提出 的 “理论质疑” 和 “步骤调整” 的方法, 在课堂上给予了有创造性欲望的学生展示的机会, 并有更 好的实验结果, 是具备榜样作用的一种展示, 并吸引了更多的学生参与到探索性和创新性的实验过 程中。当然, 在该过程中, 实验指导教师必须高效精准地引导, 防止将科学的探索方法演变成无组 织无纪律的教学实验。

\section{参 考 文 献}

[1] 孟万金. 高等教育研究, 2001, 22 (6), 58.

[2] 田曙坚, 廖一平, 焦书明. 大学化学, 2005, 20 (1), 25.

[3] 吴腊梅, 赵新筠, 张展, 胡晓允, 周忠强. 大学化学, 2021, 36 (12), 2102054.

[4] 于宇. 中国教育技术装备, 2017, No. 22, 141 .

[5] 刘廷国, 黄劲松, 郭军, 刘天宝, 汪新. 高分子通报, 2015, No. 8, 116.

[6] 装立群, 汪建民, 刘葵. 中国教育技术装备, 2012, No. 6, 130.

[7] 何建平, 韩志型, 姜兵. 实验室研究与探索, 2005, 24 (10), 110.

[8] 许家宁, 张锁秦, 张寒琦. 基础化学实验. 北京: 高等教育出版社, 2006: 134-135.

[9] 兰州大学. 有机化学实验. 北京: 高等教育出版社, 2016: 206-207. 